\title{
Dynamic Primary-Secondary Spectrum Sharing with Cellular Systems
}

\author{
Rathapon Saruthirathanaworakun*,**, ${ }^{\dagger}$, Jon M. Peha** \\ * Carnegie Mellon University, USA **Instituto Superior Técnico, Portugal \\ Email: rsaruthi@andrew.cmu.edu, peha@cmu.edu
}

\begin{abstract}
This paper considers primary-secondary spectrum sharing when the primary is a cellular system. Sharing is allowed if primary communications can withstand additional interference, rather than if there are no primary communications. We consider cases when primary and secondary systems cooperate (cooperative sharing) and when they do not (coexistent sharing). For coexistent sharing, we have devised novel mechanisms that allow secondaries to determine whether they can transmit and at what power without causing harmful interference to the primary by querying a sensor network rather than communicating with the primary system. A sensor network observes a primary's downstream communications to estimate upstream communications, and thus when additional interference would be tolerable. We show that even in highly utilized spectrum like a cellular band, a significant amount of communications among secondaries is possible. Moreover, if primary power consumption is a consideration, coexistent sharing is roughly as effective as cooperative sharing.
\end{abstract}

\section{INTRODUCTION}

Primary-secondary spectrum sharing has the potential to substantially alleviate the growing problem of spectrum scarcity [1]. Most current work, e.g. [2-5], have focused on allowing secondary devices to transmit when and where the strength of primary transmissions is so weak that spectrum is considered "unused". This is the approach adopted in the TV band [5-6]. It is the easiest form of sharing, but it is not the only viable approach. In this work, we consider the opposite: secondaries transmit when and where signal strength of primary transmissions, is so strong that secondary transmissions would not cause harmful interference, i.e. interference that causes any kind of noticeable service disruption to a primary user [1].

To explore this more challenging form of primary-secondary sharing, we consider secondary transmitters sharing with the upstream transmissions of a cellular voice communications system. A similar approach could be adopted with other kinds of primary systems in which Signal to Interference plus Noise Ratio (SINR) at the receivers is sometimes larger than necessary. Even if the extent of secondary communications in the peak hour alone does not justify the complexity of sharing in this particular case, we do demonstrate the viability of sharing when and where a primary is active. Moreover, cellular utilization is typically near its peak for a few hours of the day, and far more secondary activity is possible the rest of the time. In addition, systems using cellular technology intended exclusively for public safety have been deployed in some countries and considered in others [7-10]. Some are voice-only, and others allow data as well. Such systems have sufficient capacity for a large emergency, but are lightly used most of the time. These may also provide opportunities for sharing.

Primary-secondary sharing can be based on cooperation or coexistence [1]. Rather than assuming coexistent sharing with unlicensed secondaries, we compare the extent to which both approaches support secondary devices, licensed or not.

In cooperative spectrum sharing, a secondary device learns how much interference it can generate through communication between primary and secondary; this requires a protocol supported by both primary and secondary systems.
In coexistent spectrum sharing, there is no explicit communications between primary and secondary, and hence no need for protocols for cooperation. This paper presents novel mechanisms through which secondary devices can determine how much interference they can generate by querying a sensor network rather than the primary system. With this novel approach, the sensor network estimates primary upstream communications from monitored primary downstream communications, and thus how much interference secondary devices can generate without causing harmful interference at the primary base station.

We envision this sensor network as part of a broader costeffective approach to coexistent sharing. A system of relatively low-cost sensors can simultaneously monitor many bands, and direct any secondary device wishing to transmit to the most appropriate band; none of this complexity must be implemented in the secondary devices.

Although cooperation supports slightly more secondary communications, we show that this slight advantage comes with a significant increase in power consumption of primary users. Thus, if secondary communications and primary power consumption are both important design objectives, coexistent sharing is roughly as effective as cooperative sharing.

Much of recent research on primary-secondary spectrum sharing has assumed that secondary devices are unlicensed, but other approaches are also possible [1]: 1) Secondary License: A regulator gives exclusive rights to one entity to operate as a secondary, and 2) Secondary Market: A primary license-holder authorizes someone to operate as a secondary, perhaps in return for payment. This is already allowed in the US [11].

This paper does not consider multiple secondary systems competing for the same spectrum. Although the concepts can be extended to the unlicensed approach, this is beyond our scope. This paper also assumes the sensor network has access to information about the primary. This happens easily with the secondary market approach, as the operator of the primary system has strong incentive to share information. In the other two approaches, the regulator might mandate information sharing [1].

The observation that primary-secondary sharing with a cellular system is possible is not new. One author of this paper [12-13] and subsequently other researchers [14-18], have considered such models under various assumptions about technology and policy. Some, [12-14], found that a significant amount of secondary communications is possible even when a cellular system is $100 \%$ utilized; others did not [18].

Most considered only the simpler case of cooperative sharing. In the exception [18], a secondary device may share spectrum with cellular transmitters in distant cells, but not in the cell in which the secondary device resides. Thus, our paper is the first to compare coexistent and cooperative sharing, and the proposed approach to coexistent sharing is novel. In addition, while primary-secondary sharing with upstream cellular transmissions increases the transmit power of cellular handsets,

† Rathapon Saruthirathanaworakun is a Ph.D. student in Carnegie Mellon-Portugal program under financial support from Fundaçâo para a Ciência e a Technologia (FCT), Portugal.

\$ All research described here was performed at CMU. Any opinions expressed are those of one or both authors, and do not necessarily reflect the views of the FCC, CMU, or IST. 
no previous work has explored the tradeoff between primary transmit power and the extent of secondary communications.

We describe the scenario and underlying assumptions utilized to investigate this approach to spectrum sharing in Section II. In Section III and Section IV, we describe how cooperative and coexistent sharing work, respectively. We present simulation results in Section V. Conclusions are discussed in Section VI.

\section{SPECTRUM SHARING SCENARIO}

This paper considers sharing in the band used for upstream transmissions of a DS-CDMA cellular system. Although interference from secondary devices does not cause harmful interference, it does increase interference at the primary base station (BS), causing mobile stations (MS) to increase their transmit power to compensate for the higher interference.

Our spectrum sharing mechanisms assume the following:

1) The primary system utilizes power control. In both upstream and downstream, the primary signal will be received with the required $\frac{E_{b}}{I_{0}}$.

2) For coexistent sharing, the sensor network has following information about the primary: a) required $\frac{\mathrm{E}_{\mathrm{b}}}{\mathrm{I}_{0}}$ at a BS, and at a MS, b) maximum transmit power of a MS, c) processing gain, d) bandwidth, e) background noise density (Items a) through e) can easily be found from the specifications of the primary system equipment.), f) upper bound on intercell interference at a BS (This might be fixed, or varying with time of day.), g) lowest possible value of path loss factor between a MS and its BS (i.e. path loss factor at edge of cell), and h) a low estimate of the parameter $f$, defined as the average over all active MS's of the ratio between noise and interference $\left(n_{i}\right)$ at a given MS, and the path loss factor between the BS and this MS (i.e. $L_{M, i}$ ). (The primary can provide this low estimate from measurement data or in some scenarios from analysis.) Further details are presented in Section IV-B.

3) For coexistent sharing, the sensor network can differentiate signals from different $B S$ 's. This might be achieved if the primary system shares knowledge of the short PseudoNoise sequence that a BS uses as its identity [19]. Alternatively, the sensor network may employ directional antennas.

4) For coexistent sharing, it is possible to determine path loss factor from a secondary device to a BS when one knows that from the BS to the device. This is generally true when the difference between upstream and downstream frequencies is small [20].

In a primary cell, if a secondary device wants to transmit, the device has to determine the maximum transmit power at which it is allowed to transmit $T_{\max }^{S}$. The device can trivially calculate $T_{\max }^{S}$ from 1) path loss factor between itself and the BS $\left(L_{B, S}\right)$, and 2) maximum interference level from its transmissions that the primary can tolerate, called Secondary Tolerable Interference Level $(S T I L)$, using $T_{\max }^{S}=S T I L / L_{B, S}$.

For cooperative sharing, a secondary device learns $L_{B, S}$ and $S T I L$ from the BS, as described in Section III.

For coexistent sharing, the device determines how strong it can transmit by asking a sensor network, consisting of fixed sensors deployed in the area where spectrum sharing is taking place. The sensor network monitors downstream transmissions from surrounding BS's. It tells the secondary device its estimate of the instantaneous STIL, and the instantaneous transmit power from the BS (i.e. $P$ ), allowing the secondary to determine $L_{B, S}$ from $L_{B, S}=R_{S} / P$ where $R_{S}$ is power of the BS transmissions received at the secondary device; see assumption 4. Details on determining STIL from $P$ are in Section IV.

In addition to the basic assumptions above, we have additional specific assumptions for quantitatively assessing the sharings: 1) There are only voice communications in the primary system $^{1}$, 2) Power control in the primary is perfect, 3) The possibility of simultaneous secondary transmissions causing significant interference to a given BS is negligible (This might hold because secondary devices transmit infrequently- e.g. when secondary systems interconnect meter readers or alarm systems- or because there is coordination among secondaries), 4) Path loss between two fixed devices is constant (In practice, fluctuations are accommodated by averaging over time), 5) Locations of all active MS's are sufficiently independent to use the Central Limit Theorem (CLT) (CLT will be applied to the distribution of $f$ parameter, as discussed further in Section IV-B), and 6) A sensor network knows mean, variance, and maximum correlation coefficient of $\frac{n_{i}}{L_{M, i}}$ (The primary can provide the sensor network these three parameters from measurement data, or in some cases, from analysis).

\section{COOPERATIVE SPECTRUM SHARING}

As described in Section II, the key to allowing secondary and primary devices to transmit simultaneously in the same spectrum is the calculation of Secondary Tolerable Interference Level (STIL). STIL is derived from background noise density $\left(n_{0}\right)$, intercell interference $\left(I_{\text {intc }}\right)$ from active MS's in other cells, and Total Tolerable Interference Level (TTIL), defined as the maximum total interference that a primary BS can tolerate from sources other than the active MS's in its cell:

$$
S T I L=\text { TTIL }-\left[n_{0}+I_{\text {intc }}\right] .
$$

STIL is determined such that a secondary device can avoid causing harmful interference to any MS in a cell. In a cell, the upstream signal most vulnerable to harmful interference comes from the MS with the smallest path loss factor $\left(L_{\text {min }}\right)$ of all $K$ active MS's. With our approach, the MS with $L_{\min }$ may be required to increase transmit power up to its maximum of $T_{\max }^{M}$. Due to power control in the cellular system, at the BS, $\frac{E_{b}}{I_{0}}$ of transmissions from the MS with $L_{\min }$ will remain at the acceptable level $\gamma=\frac{T_{\max }^{M} L_{\min } G}{W[T T I L]+(K-1) T_{\max }^{M} L_{\min }}$, where $G$ and $W$ are processing gain and bandwidth of the primary, respectively. Thus, even the most vulnerable MS is safe with this TTIL:

$$
T T I L=\frac{T_{\max }^{M} L_{\min }}{W}\left[\frac{G}{\gamma}-(K-1)\right] .
$$

The BS knows all information needed to calculate TTIL and STIL using (2) and (1). Thus, the BS can tell any secondary device how much STIL the BS can tolerate.

\section{COEXISTENT SPECTRUM SHARING}

With coexistent sharing, secondary devices must also know STIL. Without communications with the primary, the secondary cannot know $L_{\text {min }}$ or $K$, both of which are needed to determine TTIL using (2), and then STIL using (1).

\footnotetext{
The same general concepts may apply to a system carrying data as well as voice, since any CDMA system operating at less than full capacity can tolerate interference. However, data brings additional complexities. First, instantaneous utilization fluctuates more in a data system, so utilization must be averaged over some period, which should be carefully chosen. Second, correlation between upstream and downstream transmission rates in a data system is less than 1 . Thus, estimations of upstream rates made by observing the downstream will have greater uncertainty. The implications of these differences are open research questions.
} 
In coexistent sharing, the sensor network gives the secondary device a conservative estimate of STIL calculated using $n_{0}$ and an Upper Bound (UB) on $I_{\text {intc }}$, which can be provided by the primary (see Section II). What is left for getting the conservative estimate of $S T I L$, is to estimate TTIL. The sensor network conservatively estimates TTIL from 1) downstream transmit power $P$ of a $B S$, which is also determined by the sensor network (because $P$ is the power of signals sent to all $K$ active MS's in the cell, the sensor network can infer information about $K$ from $P$ ), and 2) some knowledge about the primary (see basic assumptions in Section II). We describe how the sensor network estimates $P$, and TTIL in Sub-Section $I V-A$ and Sub-Section $I V-B$, respectively.

\section{A. Estimation of Downstream Transmit Power (P)}

From the power level of downstream transmission that a sensor measures $\left(R_{i}\right)$, any sensor can trivially determine current downstream power $P$ using a path loss factor from the BS to itself $\left(L_{i}\right)$. With $P$ changing over time, determining $L_{i}$ from measurements at a single sensor is impossible, but can be achieved from the measurements of multiple sensors at multiple synchronized times. If there are $N$ sensors and each has measurements at $T$ synchronized times, in total there are $N \times T$ measurements, and $N+T$ unknowns. This can be solved as a standard least-square problem. Because sensors and the BS are fixed, the sensor network can estimate $L_{i}$ before spectrum sharing starts. Thus, $L_{i}$ estimation does not need to be rapid.

\section{B. Estimation of Total Tolerable Interference Level (TTIL)}

As with cooperative sharing, TTIL is calculated using (2). However, the number of active MS's $(K)$ is obtained not through cooperation but as a function of $P$, noise and interference from other cells that each MS i perceives $\left(n_{i}\right)$, and path loss factor between the BS and the MS $\left(L_{M, i}\right)$. In general these three factors $K, L_{M, i}$, and $n_{i}$ determine how strong $P$ is.

Downstream power $P$ is the summation of transmit powers to each MS i (i.e. $P_{i}$ ) over all $K$ active MS's. Due to power control, $\frac{E_{b}}{I_{0}}$ at each MS equals the required level $\alpha$. Thus, $\frac{E_{b}}{I_{0}}$ of MS i with path loss factor $L_{M, i}$, and noise-and-interference $n_{i}$ would be at the required level $\alpha=\frac{P_{i} L_{M, i} G}{W n_{i}+\left(P-P_{i}\right) L_{M, i}}$. By taking summation of $P_{i}$ over all $K$ MS's, and defining $f$ as the average of $\frac{\mathrm{n}_{\mathrm{i}}}{\mathrm{L}_{\mathrm{M}, \mathrm{i}}}$ across all $K \mathrm{MS}$ 's, i.e. $f \triangleq \frac{\sum_{i=1}^{K} \frac{n_{i}}{L_{M, i}}}{K}$, we get a relationship between $K$ and $P$. With the $K$-and- $P$ relationship and (2), we have the equation relating TTIL with $P$ together with parameter $f$, which accounts for uncertainty in relating $K$ with $P$ :

$$
T T I L=\frac{T_{\max }^{M} L_{\min }}{W}\left[\frac{G}{\gamma}-\left(\frac{G+\alpha}{\alpha} \frac{1}{1+W\left(\frac{f}{P}\right)}-1\right)\right]
$$

Note from (3) that TTIL is an increasing function of $L_{\text {min }}$. Thus, we can obtain a conservative estimate of TTIL by using the lowest possible value of $L_{\min }$, i.e. $L_{\min }^{L B}$, as would be appropriate if one mobile station was at the edge of the cell.

Our remaining challenge is to estimate $f$. From (3), TTIL is an increasing function of $f$ so we need a low estimate of $f$, i.e. $f_{L}$, to obtain a conservative estimate of TTIL. $f$ is the average of $K$ random variables (r.v.'s) $\frac{n_{i}}{L_{M, i}}$. Although noise may be correlated from one MS's location to another, the magnitude of this r.v. is far more a function of path loss factors from the BS to MS locations, which are likely to be independent across MS's. Thus, in cases where $K$ is large (Case 2), we could use the Central Limit Theorem (CLT) to find a conservative but fairly tight $f_{L}$. On the other hand, in cases where $K$ is so small that the CLT bound would be too low to be useful (Case 1), we can make an even more conservative assumption on $K$ for the estimated TTIL. Since this is when more sharing is possible anyway, the more conservative assumption on $K$ is less problematic: Case 1 where $K<$ a positive real threshold (Th), and we derive a deterministic estimated TTIL, and Case 2 where $K \geq T h$, and we derive an estimated TTIL from $f_{L}$ obtained by applying the CLT.

For a given $T h$, the conservative estimate of TTIL for coexistent sharing (i.e. $T T I L_{C}$ ) is the minimum from the two cases. As a result, $T T I L_{C}$ is a function of $T h$, and we choose $T h$ to maximize $T$ TI $I L_{C}$ over all.

From (2), TTIL is a decreasing function of $K$. In Case 1, actual TTIL is guaranteed to be larger than the estimated TTIL (i.e. $T T I L_{C}^{\text {Case1 }}$ ) for all $K$ if $T T I L_{C}^{\text {Case } 1}$ is calculated with $K=T h$. Thus, from (2) and $L_{m i n}^{L B}$,

$$
T T I L_{C}^{\text {Case } 1}=\frac{T_{\max }^{M} L_{\min }^{L B}}{W}\left[\frac{G}{\gamma}-(T h-1)\right] .
$$

In Case 2, TTIL, as shown in (3) increases with $f$. From (3), $L_{\text {min }}^{L B}$, and $f_{L}$, the estimated TTIL (i.e. $T T I L_{C}^{\text {Case } 2}$ ) would be

$$
\text { TTIL }_{C}^{\text {Case } 2}=\frac{T_{\max }^{M} L_{\min }^{L B}}{W}\left[\frac{G}{\gamma}-\left(\frac{G+\alpha}{\alpha} \frac{1}{1+W\left(\frac{f_{L}}{P}\right)}-1\right)\right] .
$$

;we will find $f_{L}$ by applying CLT to the distribution of $f$.

From standard normal distribution, define $\beta$ as a positive real number that makes the $\operatorname{Prob}\left\{\frac{f-E\{f\}}{\sqrt{\operatorname{Var}\{f\}}}<-\beta\right\}$ negligible, where $E\{f\}$ and $\operatorname{Var}\{f\}$ are mean and variance of $f$, respectively. $f$ corresponding to $\beta$, i.e. $f_{\text {min }}$, that makes $\operatorname{Prob}\left\{f<f_{\text {min }}\right\}$ negligible is then $f_{\min }=E\{f\}-\beta \sqrt{\operatorname{Var}\{f\}}$. Recall that $f$ is defined as sample mean of $\frac{n_{i}}{L_{M, i}}$ from $K$ active MS's. With $E\left\{\frac{n_{i}}{L_{M, i}}\right\}=\mu$, then $E\{f\}=\mu$. In addition, with $\operatorname{Var}\left\{\frac{n_{i}}{L_{M, i}}\right\}=\sigma^{2}$, and $\rho_{i, j}=$ the correlation coefficient of $\frac{n_{i}}{L_{M, i}}$ between any two MS's, $\operatorname{Var}\{f\}$ is a function of $\sigma^{2}, K$, and $\rho_{i, j}$. As $K$ grows large, $f$ will get closer to $E\{f\}$ with smaller $\operatorname{Var}\{f\}$. Hence in Case $2, f_{L} \geq 0$, which is a low estimate of $f_{\text {min }}$, is obtained with $K=T h$, and $\max _{\forall i \neq j}\left\{\rho_{i . j}\right\}$ (i.e. $\rho_{\text {max }}$ ). From the assumptions in Section II, $\mu, \sigma^{2}$, and $\rho_{\max }$ are known to the secondary. Using the $f_{L}$ and (5), we have the estimated TTIL for Case $2\left(T T I L_{C}^{\text {Case } 2}\right)$ as a function of $T h$ :

$$
\begin{gathered}
\text { TTIL } L_{C}^{\text {Case } 2}= \\
\frac{T_{\max }^{M} L_{\min }^{L B}}{W}\left[\frac{G}{\gamma}-\left(\frac{G+\alpha}{\alpha} \frac{1}{1+W\left(\frac{\max \left\{\mu-\frac{\beta \sigma}{\sqrt{T h}} \sqrt{1+(T h-1) \rho_{\max }}, 0\right\}}{P}\right)}-1\right)\right](6)
\end{gathered}
$$

Note that $T T I L_{C}$ is the minimum of the two estimated TTIL shown in (4) and (6), one of which decreases with $T h$ while the other increases with $T h$. Hence, $T T I L_{C}$ is a function of $T h$, and there is an optimum threshold $T h^{*} \geq 0$ that results in the maximum possible TTIL $_{C}$, i.e. $T h^{*}$ is the point where $T T I L_{C}^{\text {Case } 1}=T T I L_{C}^{\text {Case } 2}$. This quadratic equation has the following solution: $T h^{*}=$ 


$$
\frac{-\left[\left(1-\rho_{\max }\right) B^{2} C^{2}+2 A D\right] \pm \sqrt{\begin{array}{l}
{\left[\left(1-\rho_{\max }\right) B^{2} C^{2}+2 A D\right]^{2}} \\
+4 A^{2}\left[\rho_{\max } B^{2} C^{2}-D^{2}\right]
\end{array}}}{2\left[\rho_{\max } B^{2} C^{2}-D^{2}\right]}
$$

, where $A=\frac{G+\alpha}{\alpha}, B=\frac{W}{P}, C=\beta \sigma$, and $D=1+\mu B$.

\section{NUMERICAL RESULTS}

In Sub-Section $V$ - $A$, we evaluate and compare the extent to which secondary communications are possible with coexistent, and cooperative sharing. We then quantify the impact of secondary transmissions on the primary system's transmit power in Sub-Section $V$-B. In Sub-Section $V$ - $C$, we study tradeoffs between $S T I L$ and secondary data rate, vs. transmit power of the MS's.

Results were obtained from computer simulations using the following assumptions: 1) Cell layout of the primary system is a highway model (All cells have the same radius, and are deployed along a straight line), 2) MS's are randomly placed with uniform distribution along the highway, 3) Secondary devices are located on the highway, 4) Secondary devices operate in all neighboring cells to the cell of interest, using the coexistent sharing (This assumption allows us to fairly compare the efficiency of cooperative and coexistent spectrum sharing. However, this assumption would make secondary communications achievable from cooperative sharing higher than what it would be if spectrum sharing in neighboring cells is based on cooperation), 5) All antennas are omni-directional, 6) The primary system uses load balancing, allowing number of active MS's in a channel to differ from those in other channels by no more than one, 7) Unless stated otherwise, the primary system in each cell is operating at maximum utilization, (based on Erlang-B formula with $2 \%$ call blocking probability), 8) Unless stated otherwise, the secondary is transmitting as much as possible. Unless stated otherwise, the $95 \%$ confidence interval is within $\pm 5 \%$ of the presented numbers.

Base case assumptions, including distance between the secondary transmitter and the primary base station, and distance between the secondary transmitter and the secondary receiver, are summarized in Table 1. In addition, transmission rates presented in the paper are at the Shannon limit.

Table 1 Parameter for Base Case

- Cell radius $=8 \mathrm{~km}^{2}$

- Distance between a secondary transmitter and a given base station (BS) =

$85 \%$ of cell radius

- A secondary transmitter and a secondary receiver are 400-m apart

- Center frequency: $880 \mathrm{MHz}^{3}$

- Maximum antenna gain at the $\mathrm{BS}=18 \mathrm{dBi}^{4}$

- Antenna gain at a mobile station (MS), and a secondary device $=0 \mathrm{dBi}$

- Effective gain-and-loss between a BS and a MS $=3.44 \mathrm{~dB}^{5}$

- Channel bandwidth $(W)$ and allocated spectrum of the primary are $5 \mathrm{MHz}$ and $10 \mathrm{MHz}$, respectively

- Processing gain $(G)$ for 5-MHz channel = 512 [22]

- Capacity per channel of the primary for $5-\mathrm{MHz}$ channel $=131$ active MS's ${ }^{6}$

- Maximum transmit power of a mobile station $=24 \mathrm{dBm}$ [22]

- Required $\frac{E_{b}}{I_{0}}$ at a BS $(\gamma)$, and at a MS $(\alpha)=4.5 \mathrm{dBm}[22]$

- Background noise density $\left(\mathrm{n}_{0}\right)=-174 \mathrm{dBm} / \mathrm{Hz}$ [22]

- Path loss coefficient = 3

- $99.9 \%$ of the time, primary transmissions would be at the acceptable level

\footnotetext{
${ }^{2}$ From [21, Table 3], our estimate for typical cell radius ranges from 1 to $15 \mathrm{~km}$ ${ }^{3} \mathrm{http}$ ://wireless.fcc.gov/services/index.htm?job=service bandplan\&id=cellular

${ }^{4} \mathrm{http}: / /$ www.globalsources.com/manufacturers/Base-Station-Antenna.html

${ }^{5}$ Due to antenna, connector, and miscellaneous losses; adjusted from [21, Table 3].

${ }^{6}$ Adjusted from [23, pp. 131]
}

A. Amount of Achievable Secondary Transmissions, and Performance of STIL Estimation

Fig. 1 shows the performance of the STIL estimation, described in Section IV, by comparing mean STIL from coexistent sharing with that from cooperative sharing which is the actual STIL that the BS can tolerate. This STIL estimate is conservative when there is only one or two active MS in the channel; the difference between the actual and the estimated STIL where there is only one MS can exceed eight orders of magnitude. However, this difference is less than an order of magnitude when the number of active MS's exceeds three.

In practice, the number of active MS's fluctuates as calls begin and end. We assumed that this number is modeled with an Erlang-B distribution. (See assumptions for numerical results.) Fig. 2 shows mean STIL as a function of primary utilization. As would be expected from Fig. 1, the difference between the STIL estimated with coexistent sharing and the actual value from cooperative sharing is larger with low utilization, but the difference is far less pronounced. With coexistence, mean STIL is significant until utilization approaches $100 \%$.

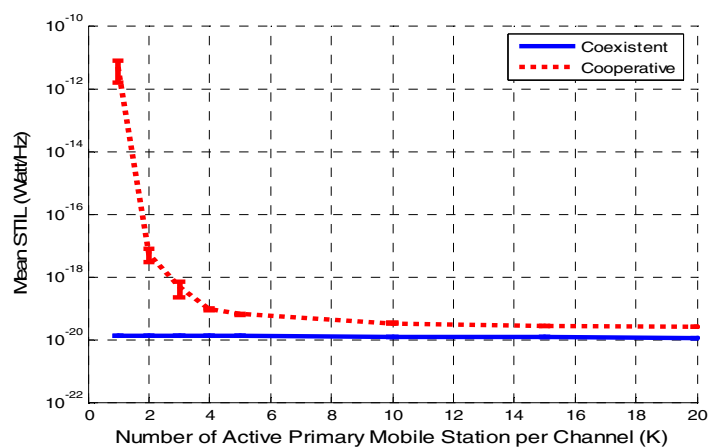

Figure 1 Mean STIL with 95\% Confidence Interval vs. Number of Active Primary Mobile Stations per Channel

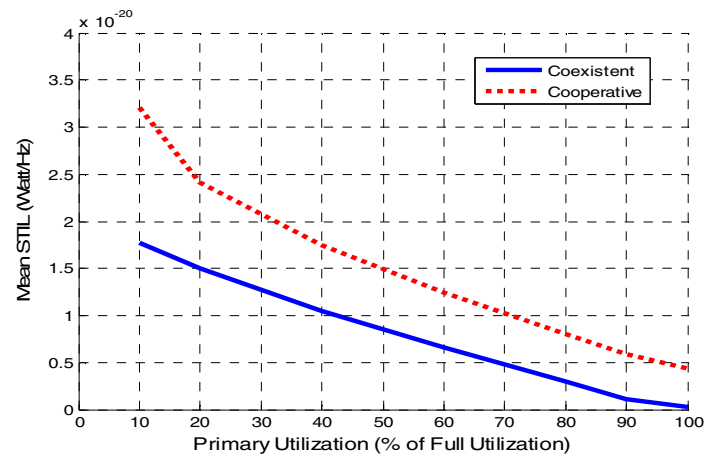

Figure 2 Mean STIL vs. Primary Utilization

Another meaningful way to assess the two sharing approaches is by quantifying the achievable data rate of secondary devices. Using distances from the secondary transmitter to the BS and the secondary receiver in Table 1, Fig. 3 shows mean secondary data rate as a function of primary utilization. There is little difference between cooperative and coexistent sharing in achievable data rates. Secondary data rate with coexistence is only around $0.1 \mathrm{bps} / \mathrm{Hz}$ less than that the rate with cooperation for the entire range of primary utilization considered.

When primary utilization is low, as would often be the case outside the busy hour, secondary data rates can be high, e.g. around $4 \mathrm{bps} / \mathrm{Hz}$ at $10 \%$ utilization, and around $0.5 \mathrm{bps} / \mathrm{Hz}$ at $50 \%$ utilization. Even when the primary is $100 \%$ utilized, the secondary can still achieve a modest amount of 
communications; $0.01 \mathrm{bps} / \mathrm{Hz}$ and $0.03 \mathrm{bps} / \mathrm{Hz}$ for sharing with coexistence and with cooperation, respectively.

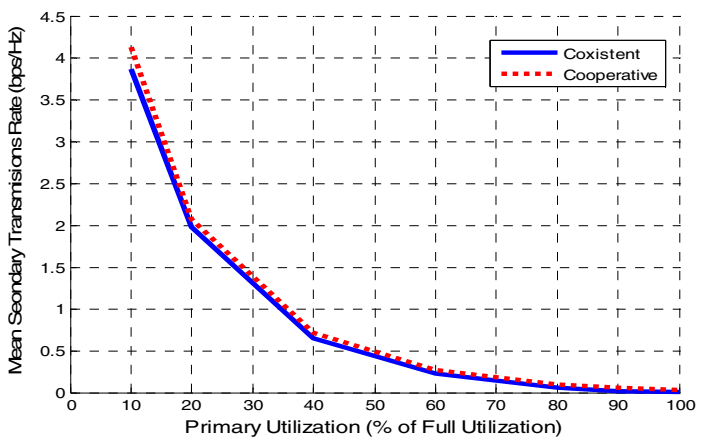

Figure 3 Mean Secondary Transmission Rate vs. Primary Utilization

\section{B. Transmit Power of a Mobile Station}

As described in Section II, to compensate for higher interference from spectrum sharing, a mobile station (MS) must increase transmit power. These differences can be significant, as demonstrated in Fig. 4, which shows the mean power at which a MS transmits with and without spectrum sharing, assuming that a secondary would transmit at the maximum level allowed.

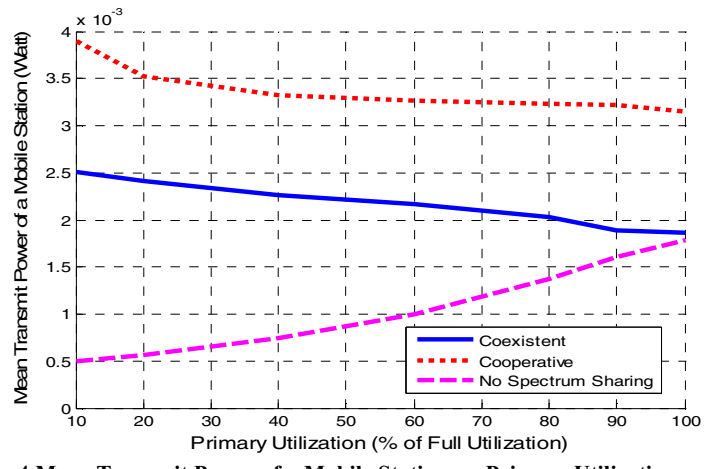

Figure 4 Mean Transmit Power of a Mobile Station vs. Primary Utilization

Without sharing, mean transmit power increases with utilization. With sharing, the opposite is true. This occurs because sharing forces the furthest MS in the cell to transmit at maximum power, and when utilization is low that MS is more likely to be close to the base station (BS). As a result, the difference in mean MS transmit power is smaller when utilization is higher. With cooperation, at $10 \%$ primary utilization, mean transmit power of a MS could be as high as eight times of that when there is no sharing, versus around two times when primary utilization is $100 \%$. With coexistence, this effect is much smaller. Indeed, at $100 \%$ utilization, coexistent sharing has little impact on primary devices.

\section{Secondary Transmissions vs. Transmit Power of a MS}

We have shown that secondary devices are often able to transmit at significant power levels without causing harmful interference, but this increases the mean transmit power of cellular MS's. Practically, the secondary does not need to generate interference as high as $100 \%$ of STIL as assumed in the previous sub-section. This sub-section shows the effect of limiting the interference generated by the secondary to less than STIL for both coexistent and cooperative sharing.

We considered four simple approaches to limit interference caused by the secondary: 1) allowing the secondary to generate interference at STIL if and only if downstream power $(P)$ is below a threshold, 2) allowing the secondary to generate interference at
STIL if the STIL is less than a threshold, and at the threshold otherwise, 3) allowing the secondary to generate interference at a fixed percentage of STIL, and 4) allowing the secondary to generate interference level at STIL if and only if $P$ exceeds a threshold. We adopted the third approach (denoted as \%STIL-L) for this paper, because among the four, at any level of primary utilization, it gives the highest secondary transmission rate with low variance.

For the \%STIL-L approach, increasing the percentage increases secondary transmit power and data rate, and primary transmit power. This tradeoff is demonstrated in Fig. 5, which is obtained by varying the fixed percentage. Fig. 5(a) shows the data rates achieved by secondary devices as a function of mean transmit power of a primary MS, and 5(b) shows mean secondary transmit power as a function of mean transmit power of a primary MS.

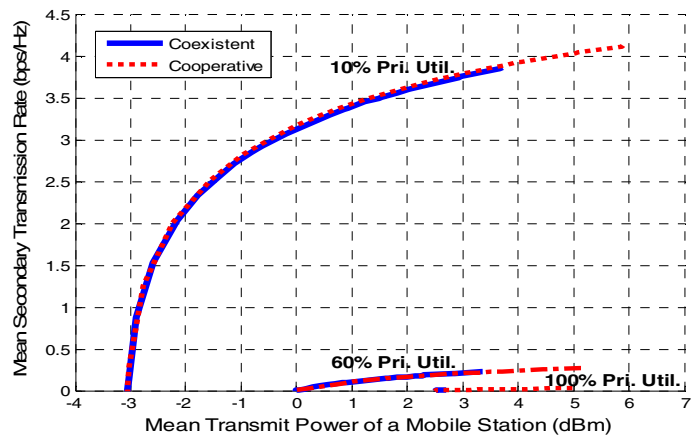

(a)

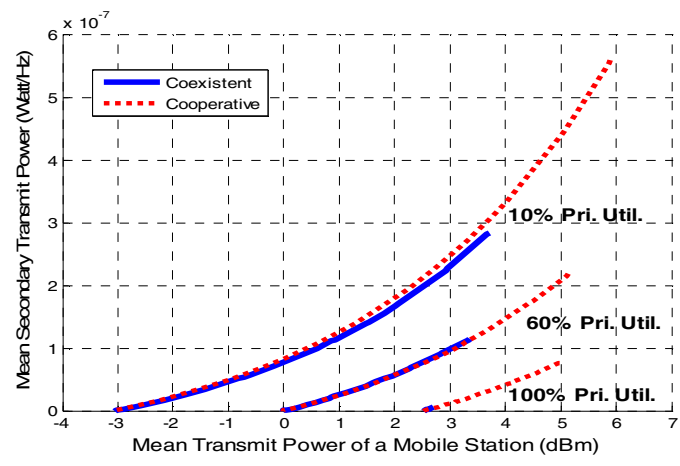

(b)

Figure 5 (a) Mean Secondary Transmission Rate, and (b) Mean Secondary Transmit Power vs. Mean Transmit Power of a Mobile Station [Trade-off, \%-STIL-L]

With both forms of spectrum sharing, Fig. 5(a) shows that allowing secondary devices to transmit at modest power levels can at first yield significant data rates, with relatively little impact to the primary. As the transmit power of secondary devices increases further, the secondary system gains less in improved data rate for each unit increase of mean transmit power of the MS. This shows a diminishing return which is much stronger when primary utilization is low than when the utilization is high. Thus, if reducing the transmit power of primary MS's is important, there are good reasons to design secondary devices that transmit at levels below the maximum allowable power.

Once designers decide to reduce the secondary transmit power to benefit the primary, there is virtually no difference between coexistent and cooperative sharing. Fig. 5(b) shows that, for a given mean transmit power of a primary MS, cooperation can enable only slightly greater transmit power for secondary devices. As shown in Fig. 5(a), this yields no 
discernable difference between coexistent and cooperative sharing in achievable data rate for the secondaries.

Cooperation does allow secondaries to transmit at a greater power. However, due to the diminishing return, the resulting gain in secondary data rate is relatively small, while the impact on the primary system is relatively large. For example, at $10 \%$ primary utilization, the maximum secondary data rate achieved by coexistent spectrum sharing is around $3.8 \mathrm{bps} / \mathrm{Hz}$ while the maximum data rate achieved by cooperative sharing is only around $8 \%$ more. However, this requires a mobile station to increase its mean transmit power by more than $50 \%$.

We define the marginal benefit of spectrum sharing as mean derivative of secondary transmission rate with respect to mean transmit power of a MS; this is the slope of the curves in Fig. 5(a). From Fig. 5(a), this marginal benefit is greater when primary utilization is lower; this is another reason why spectrum sharing may be more attractive in off-peak hours.

\section{CONCLUSIONS}

This paper studies primary-secondary spectrum sharing when the primary is a cellular system. Sharing is allowed if primary communications can withstand additional interference, rather than if there is no primary communications. We consider both the case when the sharing could happen with communications with the primaries, i.e. cooperative spectrum sharing, and without, i.e. coexistent spectrum sharing.

In coexistent sharing, secondaries query a sensor network which observes primary downstream communications to estimate how much additional interference the primary can tolerate. Our numerical results show that these estimates are conservative when there is only one or two primary devices transmitting, but are reasonably accurate with more devices.

Some argue for models based on cooperation because they can yield greater performance. However, coexistence has advantages. It works with legacy systems not designed for cooperation, and when there is no strong trust relationship between primary and secondary, as might occur if it is the regulator rather than the license-holder that gives the secondary permission to operate.

Moreover, even in highly utilized spectrum like a cellular band, a significant amount of communications among secondaries is possible. We see modest secondary data rates when the primary cellular system is at $100 \%$ utilization: around $0.01-0.03 \mathrm{bps} / \mathrm{Hz}$, for a 400 -meter link. However, real primary systems would be at $100 \%$ utilization for a few hours per day. The secondary data rates increase drastically with decreasing primary utilization. E.g. the data rate increases to $0.5 \mathrm{bps} / \mathrm{Hz}$, and $4.0 \mathrm{bps} / \mathrm{Hz}$ when primary utilization is at $50 \%$, and $10 \%$, respectively.

If we are only concerned with the amount of communications, then cooperation always yields more secondary communications than coexistence. However, increasing secondary communications also causes primary mobile stations to transmit at greater powers, especially when primary utilization is low.

If the transmit power of mobile stations is also an important design objective, we see little benefit to cooperation. Cooperation allows secondaries to transmit at even greater powers, but marginal improvements in secondary communications diminish as secondary transmit power increases. Thus, benefits of cooperation over coexistence are small, but the increased power consumption in primaries is significant. The decision of whether to choose cooperation or coexistence should be based on non-performance factors which are beyond the scope of this paper.

More generally, these results demonstrate the potential value of primary-secondary sharing in which secondaries transmit when received signal from the primary is strong, rather than when it is weak. This approach is worth considering, regardless of whether the primary happens to be a cellular system. In addition, this work shows the potential value of sharing models in which the secondary system has information about the primary, but does not cooperate with the primary in real time. Such arrangements are not typically considered today.

\section{REFERENCES}

[1] J.M. Peha, "Sharing spectrum through spectrum policy reform and cognitive radio," Proc. IEEE Special Issue on Cognitive Radio, vol.97, no.4, pp.708-19, Apr. 2009

[2] R. Tandra, A. Sahai, and S.M. Mishra, "What is a spectrum hole and what does it take to recognize one?" Proc. IEEE, vol.97, no.5, pp.82448, May 2009.

[3] J. Unnikrishnan, and V.V. Veeravalli, "Cooperative sensing for primary detection in cognitive radio," IEEE Tran. of Selected Topics in Signal Processing, vol.2, no.1, pp.18-27, Feb. 2008.

[4] M. McHenry, E. Livsics, T. Nguyen, and N. Majumdar, "XG dynamic spectrum sharing field test results," IEEE DySPAN, pp.676-84, Apr. 2007.

[5] US FCC, "In the matter of unlicensed operation in the TV broadcast bands", 2nd Report and Order and Memorandum Opinion and Order, ET Docket No. 04-186, Nov. 14, 2008.

[6] C. Stevenson, G. Chouinard, L. Zhongding, H. Wendong, S. Shellhammer, and W. Caldwell, "IEEE 802.22: The first cognitive radio wireless regional area network standard," IEEE Communications Magazine, vol.47, no.1, pp.103-8, Jan. 2009.

[7] C. Schneider, "Cape Town's TETRA public safety digital trunked radio system," Elektron, vol.19, no.4, pp.30-2, Apr. 2002.

[8] P. R. Tattersall, "Professional mobile radio. The BT Airwave public safety service and the path for technology and service evolution," $B T$ Technology Journal, vol.19, no.1, pp.142-8, Jan. 2001.

[9] J.M. Peha, "Fundamental reform in public safety communications policy," Federal Commun. Bar Journal, 59(3), pp.517-45, Jun. 2007.

[10] US FCC, "In the matter of implementing a nationwide, broadband, interoperable public safety network in the $700 \mathrm{MHz}$ band," 3rd Further Notice of Proposed Rulemaking, PS Docket No. 06-229, Sep. 2008.

[11] US FCC, "Promoting efficient use of spectrum through elimination of barriers to the development of secondary markets," 2nd Report and Order, WT Docket 00-230, Sep. 2004, footnote 237.

[12] S. Panichpapiboon, and J.M. Peha, "Providing secondary access in a cellular network," Int'l Conf. Wireless Networks, pp.591-7, June 2003.

[13] J.M. Peha, and S. Panichpapiboon, "Real-time secondary markets for spectrum," Telecommunications Policy, vol.28, no.7-8, pp.603-18, Aug. 2004

[14] H. Lee, K. Han, Y. Hwang, and S. Choi, "Opportunistic band sharing for point-to-point link connection of cognitive radios," IEEE CrownCom, pp.1-6, June 2009

[15] O. Bakr, M. Johnson, B. Wild, K. Ramchandran, "A multi-antenna framework for spectrum reuse based on primary-secondary cooperation," IEEE DySPAN, pp.1-5, Oct. 2008.

[16] M.G. Khoshkholgh, K. Navaie, and H. Yanikomeroglu, "Achievable capacity in hybrid DS-CDMA/OFDM spectrum-sharing," to appear in IEEE Transaction on Mobile Computing.

[17] A. Attar, M.R. Nakhai, and A.H. Aghvami, "Cognitive radio game: a framework for efficiency, fairness and QoS guarantee," IEEE ICC, pp.4170-4, May 2008.

[18] E.G. Larsson and M. Skoglund, "Cognitive radio in a frequency-planned environment: Some basic limits," IEEE Tran. On Wireless Communications, vol.7, no.12, pp.4800-06, Dec. 2008.

[19] S. Willenegger, "cdma2000 physical layer: an overview," Journal of Communications and Networks, vol.2, no.1, pp.5-17, March 2000.

[20] E. Perahia and D. Cox, "Shadow fading correlation between uplink and downlink," IEEE VTC, pp.308-12, Spring 2001.

[21] S. Newman, "Comments to the FCC in the matter of service rule for the 698-746, 747-762 and 777-792 MHz bands," WT Docket 06-150, Sep. $29,2006$.

[22] M.A. Abu-Rgheff, Introduction to CDMA Wireless Communications, Academic Press, 2007.

[23] Technical Staff, Bell Labs, Handbook of CDMA System Design, Engineering, and Optimization, Kyoung Il Kim Ed., Prentice Hall, 1999. 